\title{
A system for electrotactile feedback using electronic skin and flexible matrix electrodes: Experimental evaluation
}

\author{
Marta Franceschi, Lucia Seminara, Member, IEEE, Strahinja Dosen, Member, IEEE, Matija \\ Strbac, Maurizio Valle, Member, IEEE, and Dario Farina, Senior Member, IEEE
}

\begin{abstract}
Myoelectric prostheses are successfully controlled using muscle electrical activity, thereby restoring lost motor functions. However, the somatosensory feedback from the prosthesis to the user is still missing. The sensory substitution methods described in the literature comprise mostly simple position and force sensors combined with discrete stimulation units. The present study describes a novel system for sophisticated electrotactile feedback integrating advanced distributed sensing (electronic skin) and stimulation (matrix electrodes). The system was tested in eight healthy subjects who were asked to recognize the shape, trajectory and direction of a set of dynamic movement patterns (single lines, geometrical objects, letters) presented on the electronic skin. The experiments demonstrated that the system successfully translated the mechanical interaction into the moving electrotactile profiles, which the subjects could recognize with a good performance (shape recognition: $86 \pm 8 \%$ lines, $73 \pm 13 \%$ geometries, $72 \pm 12 \%$ letters). In particular, the subjects could identify the movement direction with a high confidence. These results are in accordance with previous studies investigating the recognition of moving stimuli in human subjects. This is an important development towards closed-loop prostheses providing comprehensive and sophisticated tactile feedback to the user, facilitating the control and the embodiment of the artificial device into the user body scheme.
\end{abstract}

Key words - Tactile Devices, Human-Computer interaction, Prosthetics

\section{INTRODUCTION}

A myoelectric prosthesis can be used to compensate for the loss of motor function after a hand amputation. In this system, the electrical activity of the user's muscles is recorded, processed and then decoded to estimate the user intention. Decoding is performed using simple heuristics, as in the classic two-channel control, or machine learning (pattern classification and regression) in multichannel systems [1], [2]. Typically, the same muscles that were used to accomplish a motor task before the amputation control analogous functions in the prosthesis, e.g., hand and wrist flexors and extensors control prosthesis closing and opening, respectively. This association provides an intuitive connection between the brain and the prosthesis, but this connection is unidirectional. However, a bilateral

- Marta Franceschi, Lucia Seminara and Maurizio Valle are with the Department of Electrical, Electronic, Telecommunications Engineering, and Naval Architecture (DITEN), University of Genoa, Italy. E-mails: marta.franceschi@edu.unige.it; lucia.seminara@unige.it; maurizio.valle@unige.it.

- Strahinja Dosen is with the Biomedical Engineering Group, Clinic of Trauma Surgery, Orthopaedic Surgery and Plastic Surgery, University Medical Center Göttingen (UMG), Georg-August University, Göttingen, Germany. E-mail: strahinja.dosen@bccn.uni-goettingen.de.

- Dario Farina is with the Department Bioengineering, Imperial College London, London, UK. E-mail: d.farina@imperial.ac.uk.

- Matija Strbac is with Tecnalia Serbia Ltd., Belgrade, Serbia and the University of Belgrade - School of Electrical Engineering, Belgrade, Serbia. E-mail:matija.strbac@tecnalia.com communication between the brain and the periphery, including both efferent and afferent information flow, is necessary for the human movement learning and execution [3]. Closing the prosthesis control loop by providing sensory feedback to the user is a key point in research on active prosthetics [4], [5] as well as an often cited requirement of the prosthesis users [6]. Nevertheless, apart from one recent example [7], there are still no commercially-available prostheses transmitting somatosensory information back to the user.

Artificial tactile feedback can be provided using a method known as sensory substitution [8]. The prosthesis is equipped with sensors measuring the system state (e.g., joint angles) as well as interaction with the environment (e.g., grasping force). To restore the feedback, the sensor data are read online, translated into stimulation profiles according to the selected coding method and the stimulation is delivered to the user invasively (e.g., neural [9] and brain stimulation [10]) or non-invasively (e.g., electro [11] and vibrotactile interfaces [12]). The information is transmitted by modulating the stimulation parameters. In electrotactile stimulation, low-level electrical current pulses are delivered to the skin to depolarize skin afferents, thereby eliciting tactile sensations. 
The fidelity of the feedback that can be provided to the user depends on the available sensors and stimulation interfaces. Typically, current prostheses are equipped with only basic position and force sensors [13]. Importantly, some more sophisticated sensing elements are becoming available. BioTac [14] is a sensorized finger equipped with a matrix of pressure sensors across the fingertip, as well as a vibration and temperature sensor. Electronic skins integrating matrices of tactile (and other) sensing elements embedded into flexible structures have been fabricated (eg [15]-[19], references in [20]).

Most of these sensors were originally developed in the robotic framework, to provide tactile feedback for automatic robot control. However, these highly sophisticated sensing interfaces can as well be utilized in prosthetics, both for control and to transmit somatosensory feedback to the user, analogously to the manner natural skin is exploited by the human nervous system during grasping and manipulation [21]. An electronic skin (e-skin) can be therefore integrated into a prosthetic device to endow the prosthesis with artificial cutaneous sensing by electromechanically converting touches into electrical signals to be delivered to the human subject. The information from multiple contact points can be used by the embedded prosthesis controller to automatically evaluate the grasp stability, control grip formation and/or prevent slipping [22]. In the context of feedback to the user, such sensors are comprehensive sources of tactile signals, which can be utilized to provide an unprecedented level of afferent inflow, facilitating thereby the integration of the prosthetic device and the user body and mind, as proposed conceptually in [23][25], and stimulating the cognitive and psychological mechanisms related to body ownership [26]. Some recent sensors also implement biologically-inspired coding of the tactile information. For example, [27] describes a flexible electronic skin capable of quantifying the applied pressure and generate electrical signals to directly deliver such a sensory input in the form of electrical pulse trains mimicking nerve action potentials to a living nerve cell of the mouse brain.

Regarding the stimulation interfaces, non-invasive methods are still most common [5]. Most studies [28]-[30] considered single stimulation units with parameter modulation (e.g., frequency and intensity proportional to the feedback variable value) and a few [31]-[33] evaluated multichannel approaches using several electrodes or vibrators allowing for the spatial coding (e.g., stimulation location communicates the feedback variable). The studies provided important insights regarding the role and advantages of feedback in prosthetics, but they utilized simple force and position sensors embedded into the prosthesis. These conventional stimulation configurations are not suitable for interfacing with the aforementioned advanced sensing systems. In order to transmit the information from a large number of sensing elements, available in the e-skin systems, the electrotactile interface needs to implement many stimulation channels integrated into a compact device.

In this manuscript, we present a first prototype of such a distributed sensing and stimulation interface and we evaluate the feasibility of communicating the tactile information to a human subject. The system comprises an e-skin including an array of sensing elements (taxels), acquisition electronics and multichannel stimulator connected to flexible matrix electrodes placed on the forearm.

Although the concept of applying e-skin technology for somatosensory feedback in prosthetics has been proposed earlier [5], the present study is the first evaluation of an online system implementing the prospective communication link, i.e., the information transmission from e-skin to the human subject.

\section{Materials and Methods}

\subsection{System Architecture}

The tactile feedback system comprises the following components (Fig. 1): 1) e-skin with 64 sensing elements (taxels), 2) custom-made electronics for the signal conditioning, 3) data acquisition card (NI cDAQ 9174, National Instruments, USA), 4) fully programmable multichannel electrotactile stimulator (MaxSens, Tecnalia, Spain) connected to two flexible matrix electrodes, and 5) a host laptop PC. The host PC runs the online control loop which was implemented in Matlab 2013b and operated as follows. The e-skin converted mechanical information (i.e. a specific way of touching) into a set of electrical signals (one signal per sensor). The taxel signals were sampled at the frequency of $1 \mathrm{kHz}$ and the data were segmented into intervals of $50 \mathrm{~ms}$ for processing (as described below). The stimulation parameters were sent to the stimulator, which delivered the stimulation. The stimulation parameters were therefore refreshed at $20 \mathrm{~Hz}$ (1/data window length).

The e-skin (Fig. 1b) was a rectangular patch $(12.8 \times 4.8$ $\mathrm{cm}$ ) made of Polyvinylidene Fluoride (PVDF), which is a piezoelectric polymer directly converting mechanical stress into charge [34]. The intrinsic flexibility of this polymer in the form of a film together with its wide frequency bandwidth $(1 \mathrm{~Hz}-1 \mathrm{kHz})$ and large measurable pressure 
range (50 Pa-1 MPa) make it a good candidate as functional constituent of a flexible e-skin measuring dynamic contacts. Sixty-four different sensors (taxels) were obtained by screen printing patterned electrodes [35] on a single commercial $100-\mu \mathrm{m}$ thick PVDF sheet [36]. Taxels were circular (radius $1.5 \mathrm{~mm})$ and arranged in a rectangular array $(9.6 \times 3.5)$, with the sensor pitch (centre-to-centre distance) of approximately $8 \mathrm{~mm}$. In the present application, the PVDF sheet was placed on a rigid substrate and an elastic layer (Polydimethylsiloxane) was added on top for stress transmission and sensor protection [37]. The charge is read from each taxel by a custom-made multi-channel charge amplifier (CA), converted into voltage and conditioned by a band-pass filter (details are reported in [38]). The analog outputs from the amplifier were connected to the analog inputs of the data acquisition card, which sampled the signals at $1 \mathrm{kHz}$ and communicated the data to the host PC via a USB port.

The MaxSens stimulator is a clinical prototype developed by Tecnalia Research and Innovation for the implementation and assessment of the electrotactile feedback. The system was presented previously and tested in the context of transmitting multimodal proprioceptive [39] and high-resolution force feedback [40], facilitating the closed-loop control of a myoelectric prosthesis. The MaxSens is actually the next generation of the IntFES stimulation system [41], adjusted for electrotactile feedback applications (lower stimulation amplitudes with higher resolution).

The MaxSens comprises a single stimulation unit that can generate biphasic symmetric current-controlled pulses and distribute these pulses in time and space over the multi-pad electrode, thus, providing multichannel stimulation interface. The stimulator is fully programmable and the stimulation parameters can be adjusted online by sending text commands from the host PC via a Bluetooth connection. The current amplitude can be changed in the range $0-5 \mathrm{~mA}$ in increments of $0.1 \mathrm{~mA}$, the pulse width from 50 to 1000 us in increments of 10 us, and the frequency resolution is $1 \mathrm{~Hz}$ with the maximum rate of $400 \mathrm{~Hz}$. The output channel of the stimulator was connected to a demultiplexer that can route the generated pulse to one of 64 stimulation channels. Importantly, the firmware implements the switching protocol while the user (host PC) needs only to set the stimulation frequency (common to all channels) and intensity (amplitude and/or pulse width) independently for the chosen active channels.

The stimulator was connected to two flexible matrix electrodes (Fig. 1c). These electrodes were custom designed, each array consisting of 16 round electrodes with the radius of $0.5 \mathrm{~cm}$, arranged in a $4 \times 4$ geometry, with the total size of $10.4 \times 9.1 \mathrm{~cm}$. The distance between two adjacent electrodes was approximately $1.5 \mathrm{~cm}$, which is well above the two-point discrimination threshold for electrotactile stimulation ( $9 \mathrm{~mm}$ on the forearm [42]). The round electrodes were the cathodes whereas the long bars in-between the cathodes served as the common anodes. It should be noted that the stimulation pulses were biphasic with symmetrical compensation, so that the current changed direction between the stimulus and the compensation pulse of the same width and amplitude. In this context, the terms anode and cathode refer to the physical design of the electrode and the consequent role of the pad (i.e., depolarizing vs. neutral) rather than polarity (i.e., electrical current direction). Namely, the surface of the anode pads was more than 10 times larger than the surface of the cathodes. The anodal current densities were therefore significantly lower and below the threshold required to excite the cutaneous afferents. Consequently, the tactile sensations were produced only below the cathodes, as confirmed by the psychometric assessment [43]. This configuration was selected in order to localize the current flow, from a cathode to its closest anode segment, eliciting focused tactile sensation.

The stimulation electrodes of custom design were produced in the process of screen-printing using medical grade conductive ink on the flexible foil. Basically, the electrodes consist of a polyester film substrate, an $\mathrm{Ag} / \mathrm{AgCl}$ conductive layer, and an insulation coating covering the conductive leads. $\mathrm{Ag} / \mathrm{AgCl}$ was used as an active material for the electrodes because of the low half-cell potential of approximately $220 \mathrm{mV}$ and the low electrode impedance [44]. To improve the electrode-skin contact, conductive hydrogel pads (AG702, Axelgaard, DK) were placed on the top of electrode pads. This design allowed electrode bending and provided a close contact of the electrode with the skin. Two arrays were placed one after the other on the participant forearm (Fig. 1c), providing a total of 32 available electrode channels.

The electrodes used in the present study were fairly large, as they were based on a design that was developed and tested previously [43]. Importantly, the technology for electrode production is flexible and allows custom solutions, as explained in previous paragraph. Therefore, an important future goal will be to optimize the design of the electrode (e.g., decrease the pad size, increase pad number and density etc.).

The stimulation system was developed and tested by Tecnalia. The tests included a comprehensive assessment of subjectively perceived electrotactile sensations elicited by information. 


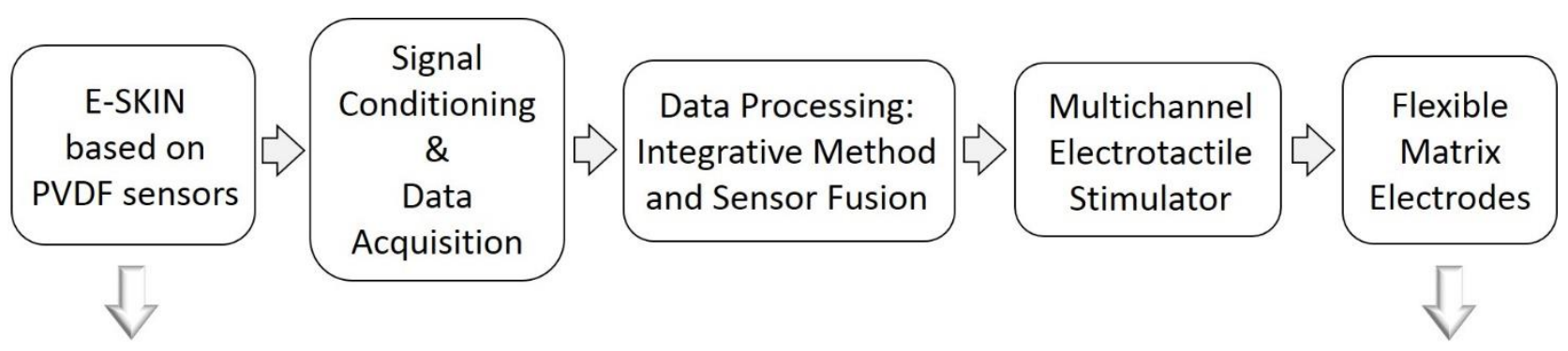

(a)

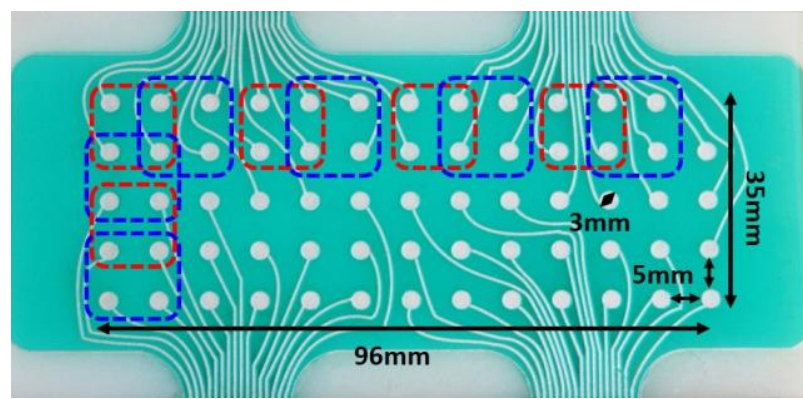

(b)

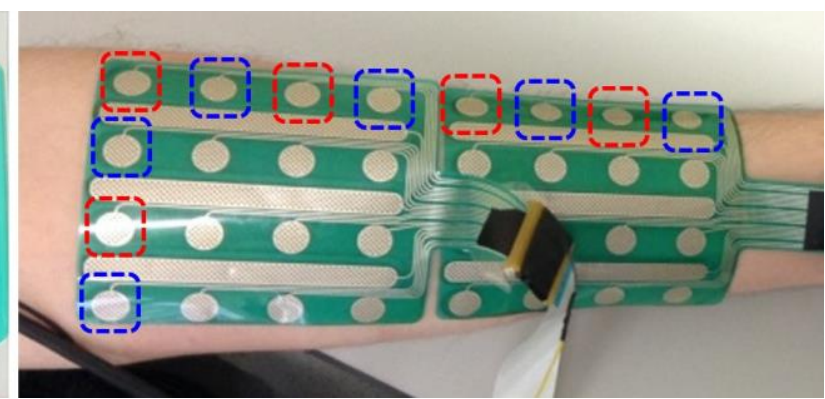

(c)

Fig. 1. (a) System Architecture. The system comprises e-skin with 64 sensing points, charge amplifier assessing the response of each taxel to mechanical stimulus, data acquisition card to sample the signals, and multichannel stimulator with flexible matrix electrodes integrating 32 electrode pads to deliver the electrotactile stimulation to the subject. (b), (c) E-skin and Electrode Array: Sensor Fusion. The taxel signals are processed using integrative method and the signals from the neighboring taxels $(2 \times 2)$ are fused in order to map more taxels to less electrode pads (red and black dashed lines).

the system [43]. In the present study, the electrode size and arrangement of the pads were modified with respect to that initial design, in order to better mimic the outline of the eskin. However, the size of the pads and the inter-pad distance were unaltered so that the quality of elicited sensations remained the same as before [43].

The intensity and quality of the electrostimulation (isolated touch, vibration, tingling) [45], [46] can be adjusted by modulating the pulse amplitude, width, and rate of delivery (parameter coding) as well as the location of stimulation by changing the active electrode (spatial coding) [11], [12].

\subsection{Tactile data processing}

Before starting the online loop, the baseline levels of the taxel signals were determined. Signals were recorded from the skin for at least $5 \mathrm{~s}$ with no mechanical interaction and the mean value of the collected samples was computed for each taxel. During the online operation of the system, the baseline was subtracted from each taxel output, centering the signals at zero level. The zero-mean taxel data were then multiplied by a calibration matrix that represented the scaling factors compensating for the variation of sensitivity across individual taxels.

The data were acquired from the skin in blocks of 50 samples corresponding to the data segments of $50 \mathrm{~ms}$. After applying the calibration steps, as described above, the integrative processing was implemented. The taxels are piezoelectric sensors, capturing the dynamic aspects of the mechanical input to the skin. Figures $2 \mathrm{a}, \mathrm{c}$ depicts the taxel signals responding to a step input in pressure, i.e., a finger pressing and then releasing the skin. As a response, the taxels generated two phasic burst outputs (Fig.2c, d), indicating the pressure and release events, while inbetween there was almost no response apart from some wiggling due to small movements of the pressing finger. The contact event was indicated with a positive deviation whereas release generated a negative deflection of the taxel signal. Also, the figure demonstrates that the mechanical stimulus propagates across the skin (Fig. 2a), activating neighboring taxels and even taxels distant with respect to the point of contact, with the response intensity decreasing with the distance.

Raw taxel data were processed in order to capture the static features of the mechanical input and also to suppress the aforementioned spurious activations. For the latter, a threshold was defined and the taxel signals below the threshold were set to zero. Considering that contact and release events elicited transient responses of opposite polarity (Fig. 2c), an integrative method was implemented to estimate the static response: a mean value within the 50ms data segment was computed for each taxel and the taxel means were added cumulatively across the time intervals. 


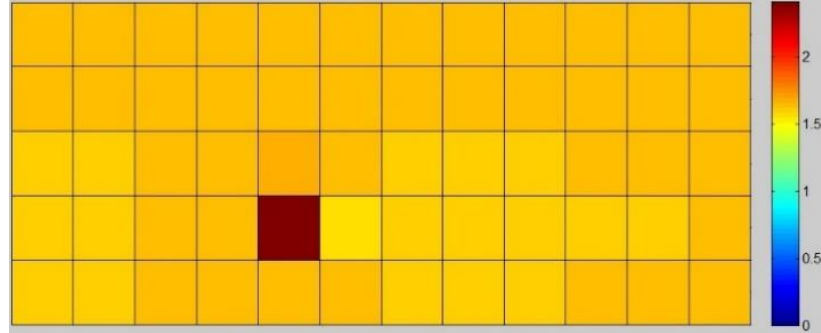

(a)
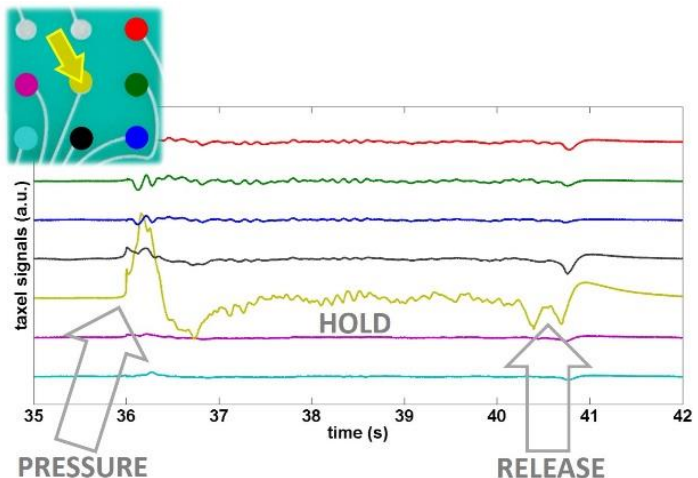

(c)

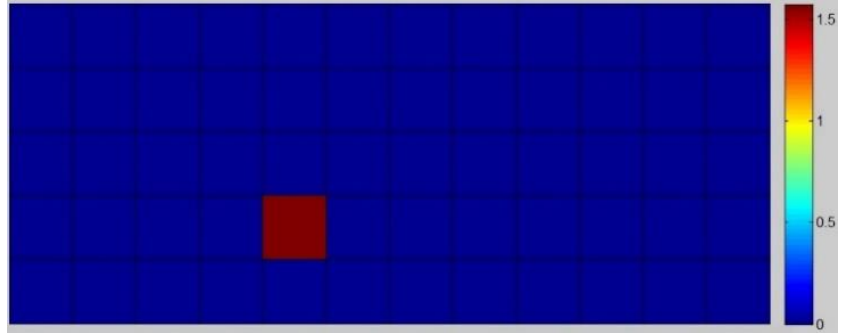

(b)

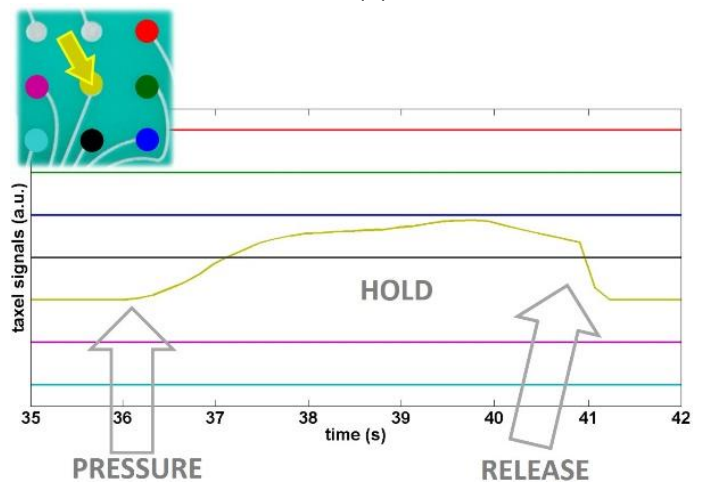

(d)

Fig. 2. Electrical response (taxel signals) due to a pressure applied to the e-skin using a finger. The mechanical stimulus comprised a step input (pressure-hold-release) delivered to a single taxel. TOP: color map representing a snapshot of all the taxel signals while pressure is applied for (a) raw and (b) processed data. BOTTOM: signal traces for the stimulated taxel (inset, yellow arrow) and its neighbors for (c) raw and (d) processed data. The signal plots have the line color of the corresponding taxels (see inset). The signals are zero-mean but where shifted using offsets for the sake of plot readability. The contact and release events elicit positive and negative deflections in the raw signals, respectively. Processed signals are clean from spurious activations and capture also the static properties of the mechanical stimulus.

More formally, let's define (i) $S_{i}(k)$ as a sequence of vectors of $\mathrm{N}=64$ elements (one element per taxel), representing taxel outputs at the time instant of $\mathrm{k}$ milliseconds, where $\mathrm{k}=1,2, \ldots 50$, within the $i$ th data segment (50-ms duration), and (ii) $\bar{S}_{i}$ as a vector $(\mathrm{N} \times 1)$ containing taxel data averaged across the $i$ th data segment:

$$
\bar{S}_{i}=\frac{1}{50} \sum_{k=1}^{50} S_{i}(k)
$$

The mean tactile data from the present data segment $\bar{S}_{i}$ were added to the cumulative mean $\bar{S}_{i-1}$ of all the previous data segments yielding:

$$
\bar{S}_{i} \leftarrow \bar{S}_{i}+\bar{S}_{i-1} \wedge \bar{S}_{0}=0
$$

The final output of this processing was a single value per taxel per data segment. The processed taxel signals are depicted in Fig. 2(b-d). The raw signals are sampled at 1 $\mathrm{kHz}$ while the processed data are outputted at the sampling rate of $20 \mathrm{~Hz}$ (new value each $50 \mathrm{~ms}$ ). During this time interval (Fig. 2c vs Fig. 2d), the signals rise as the pressure is applied, remain at the non-zero level while the pressure is maintained, and decrease back to zero as the pressure is released. The initial positive deflection (contact) increased the cumulative mean, which then remained constant during contact since the taxel signal was below the threshold. Finally, a negative deflection due to release decreased the cumulative mean. To counteract the fact that positive and negative deflections were not completely symmetric, the cumulative mean was further processed. The cumulative mean was forced to zero when below a fixed threshold until it exceeded the threshold due to a new contact event (negative values for the cumulative mean were not allowed). The response signal amplitude scaled with the applied pressure input.

\subsection{Electrotactile spatial coding}

To map 64 tactile sensor outputs to 32 stimulation electrodes, tactile data from neighboring taxels were averaged in groups of $2 \times 2$. This resulted in 32 mean values that exactly corresponded to the number of stimulation channels. The mapping between taxel groups and stimulation electrodes is shown in Fig. 1(Lower panel). The electrotactile channels were activated only if the corresponding mean value was above a predefined threshold, which has been previously chosen for each participant in pilot tests to guarantee a clear perception of the electrostimulation (details are reported in Sect. 3.2). Since the focus in the present study was on the recognition of spatially moving stimuli (see Experimental Protocol), 
the stimulation location was modulated while the intensity and frequency were kept constant. We selected the stimulation frequency of $100 \mathrm{~Hz}$ based on our previous experience [47]. A single electrical pulse elicits a brief tactile sensation (transient touch). However, at the high rates, such us $100 \mathrm{~Hz}$, the discrete sensations fuse into a continuous feeling of tingling or tickling, which can be easily and clearly perceived by the subjects. The intensity was set for each electrode and subject individually to just above the sensation threshold (see the Experimental Procedures), resulting in comfortable and localized tactile sensations.

\section{EXPERIMENTAL EVALUATION}

\subsection{Participants}

The experiments were conducted in eight healthy volunteers (three females, five males, $28 \pm 5$ years). The study has been approved by the local Ethical committee of the Specialized Hospital for Rehabilitation and Orthopedic Prosthetics (approval number 1172). Before starting, the subjects signed an informed consent form.

\subsection{Experimental Procedures}

The subjects were seated comfortably on a chair in front of a table. The forearm of the dominant arm was placed on the table surface, with the volar side oriented upwards. The electrode arrays were positioned on the volar aspect of the subject forearm. The first electrode was placed $1 \mathrm{~cm}$ below the elbow and the second right after it. The electrodes were then secured with medical tape to prevent movement and improve contact. The electrostimulation was adjusted to be strong enough so that it is clearly perceivable, but without being uncomfortable or causing pain. First, electrotactile sensation thresholds were determined for each of the 32 channels individually using the method of limits [48]. The current pulse amplitude, starting at zero, was increased in steps of $0.1 \mathrm{~mA}$ until the subjects reported that they felt the electrostimulation. After determining the subjective stimulation threshold, the perception was additionally checked by repeatedly activating/deactivating the pad, and asking the subject to report when he/she felt the stimulation. If the subject was able to detect the change in state (on/off), the stimulation amplitude was accepted as appropriate, and the procedure was repeated for the next pad. Otherwise, the amplitude was increased until the subject could detect the transition. Furthermore, small adjustments in the current amplitudes were made to roughly equalize the tactile sensations across the electrode pads. The experimenter activated the pads in sequence from the first to the last and the subject reported different from the others. In this case, the current amplitude would be increased/decreased, as needed. This was repeated several times until the subject reported that the elicited sensations across pads were indeed similar. This procedure lasted between 15 and $30 \mathrm{~min}$.

The experiment comprised blocks of different trials in which the experimenter applied a mechanical stimulus to the e-skin, while the system online translated this stimulus into stimulation profiles delivered to the subject (as described before).

The task for the subject was to perceive the stimulation and report to the experimenter the properties of the mechanical stimulus.

The mechanical stimulus was applied to the skin using a small roller to ensure contact reproducibility between different trials. To produce a natural and realistic motion, the experimenter moved the roller along the skin at a selfselected speed and pressure, aiming to be consistent across the trials.

Three categories of mechanical patterns have been tested (see Table 1 and Fig. 3): single lines (10 patterns), geometrical shapes (7 patterns) and letters (8 patterns). The lines and geometrical shapes were additionally organized in subcategories (see Table 1).

The subject could not see the experimenter, nor the skin. A sheet of paper was placed in front of the subject with a schematic drawing of the electrode array. After receiving the electrical stimulation, the subjects were first asked to verbally estimate the perceived shape of the movement: longitudinal, transversal, diagonal line; square, rectangle or triangle; an exact letter (Fig. 3). If the shape estimate was correct, the subjects were asked to retrace the perceived stimulus by moving their index finger across the schematic drawing. If this corresponded to the pathway of rolling when delivering the stimulus (and thereby applied electrical stimulation), it was deemed that the subjects correctly recognized the movement trajectory. Finally, if the subjects retraced the trajectory segments (lines) in the proper direction, as indicated by arrows in Fig. 3, the movement direction was also correctly perceived.

During the first three blocks, after the subject reported the recognized movement features, the experimenter provided feedback about the correct features of the applied movement, in terms of shape, trajectory, and direction. The experimenter verbally described and also demonstrated the specific movement using a roller (reinforced learning phase). In the last two blocks, such information was not provided to the subject (test phase). 
TABLE 1

TOUCH MODALITIES

\begin{tabular}{|c|c|c|c|c|}
\hline \multirow{34}{*}{$\stackrel{\text { 됨 }}{{ }^{\prime}}$} & \multicolumn{4}{|c|}{ LINES } \\
\hline & \multirow{13}{*}{ 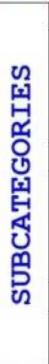 } & \multicolumn{3}{|c|}{ LONGITUDINAL } \\
\hline & & & It & Longitudinal: $T \circ p$, from left to right \\
\hline & & & Im & Longitudinal: Middle, from right to left \\
\hline & & & lb & Longitudinal: Bottom, from left to right \\
\hline & & & $\mid \mathrm{lb} /$ & Longitudinal: Bottom, from left to middle \\
\hline & & \multicolumn{3}{|c|}{ TRANSVERSAL } \\
\hline & & & $t i$ & Transversal: flrst column, from top to bottom \\
\hline & & & to & Transversal: fOurth column, from bottom to top \\
\hline & & & te & Transversal: Eighth column, from top to bottom \\
\hline & & & $t t$ & Transversal: $T$ welfth column, from bottom to top \\
\hline & & \multicolumn{3}{|c|}{ DIAGONAL } \\
\hline & & & $d r l$ & Diagonal: from bottom $R$ ight corner to top Left \\
\hline & & & $d l r$ & Diagonal: from bottom Left corner to top $R$ ight \\
\hline & \multicolumn{4}{|c|}{ GEOMETRIES } \\
\hline & \multirow{10}{*}{ 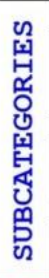 } & \multicolumn{3}{|c|}{ TRIANGLE } \\
\hline & & & bt & Bottom pointing Triangle \\
\hline & & & Itr & Left TRiangle \\
\hline & & & $r t$ & Right Triangle \\
\hline & & \multicolumn{3}{|c|}{ RECTANGLE } \\
\hline & & & $b r$ & Bottom Rectangle \\
\hline & & & $\operatorname{tr}$ & Top Rectangle \\
\hline & & \multicolumn{3}{|c|}{ SQUARE } \\
\hline & & & Is & Left Square \\
\hline & & & rs & Right Square \\
\hline & \multicolumn{4}{|c|}{ LETTERS } \\
\hline & \multirow{8}{*}{\multicolumn{2}{|c|}{ 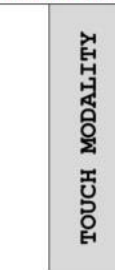 }} & $L$ & $\mathrm{~L}$ \\
\hline & & & $T$ & $T$ \\
\hline & & & $N$ & $\mathrm{~N}$ \\
\hline & & & $x$ & $x$ \\
\hline & & & $Z$ & $Z$ \\
\hline & & & $\perp$ & Mirrored T \\
\hline & & & $\Lambda$ & Mirrored V \\
\hline & & & 5 & Mirrored Z \\
\hline
\end{tabular}

Movement test patterns applied to the e-skin.

Each line in this table corresponds to a single touch modality (pattern).

$S$ (Shape), $T$ (Trajectory) and D (Direction): touch modality features.

\subsection{Data analysis}

The main outcome measure was the success rate (SR) in recognizing a feature of the movement, i.e., movement shape, trajectory and direction. The SRs for the trajectory and direction were conditioned on the correct recognition of the shape, as described above. The SRs were computed per subject for the specific categories (lines, geometries, letters) and sub-categories, i.e., shapes (lines: longitudinal, transversal and diagonal; geometries: rectangle, square and triangle) of the tested mechanical stimuli. The letters were treated differently, with no subcategories, as each letter was peculiar and different from others. The SRs of all subjects were then averaged to obtain the overall mean SR and its standard deviation. The results were reported as mean \pm standard deviation in the text and the figures.

The data were tested for normality using KolmogorovSmirnov test. In case the test indicated normal distribution, one- and two-way repeated measure ANOVA was applied to assess statistically significant differences at the level of the group followed by Tukey's honest significant difference test for post hoc pairwise comparison.

If the distribution was not normal, the Friedman test was used instead of ANOVA, while the post hoc analysis
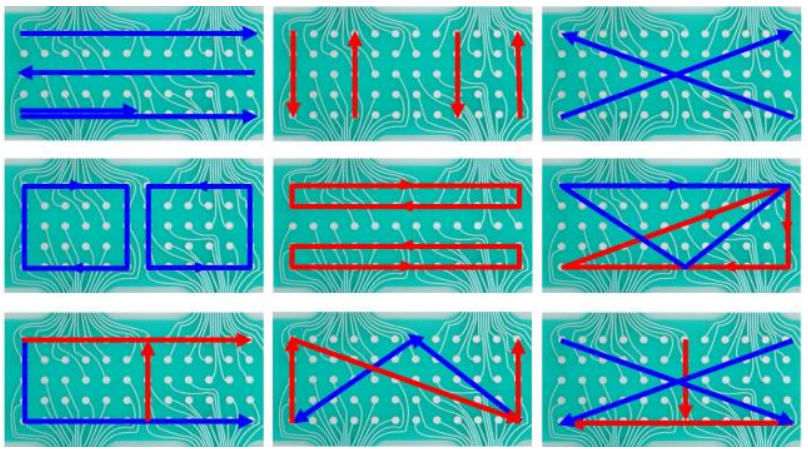

Fig. 3. Example of movement test patterns applied to the e-skin by an experimenter using a roller. TOP: Iongitudinal (left), transversal (center), diagonal (right) single lines. MIDDLE: square (left), rectangular (center), triangle (right). BOTTOM: letters: T and L (left), $\mathrm{N}$ and mirrored $\mathrm{V}$ (center), $\mathrm{X}$ and mirrored $\mathrm{T}$ (right).

remained the same. Following this scheme, one-way ANOVA or Friedman test (as appropriate) were used to compare the success rates in recognizing movement features across movement categories and sub-categories (see Table 1). Finally, two-way ANOVA could be applied only in the case of geometries (category), where the factors were subcategory (triangle, rectangle, and square) and movement feature (trajectory, direction). To evaluate the effect of training, the success rates in recognizing all the movement features simultaneously during training (blocks 1 to 3, pooled) and testing (blocks 4 and 5, pooled) were compared using two-way ANOVA with category (lines, letters, geometries) and phase (training, testing) as the factors. The threshold for the statistical significance was adopted at $p<0.05$, and the statistical analysis was conducted in Matlab R2014a (MathWorks, US).

\section{Results}

There was no significant difference in performance between the reinforced learning and testing phase. There was no significant interaction nor main effect in the twoway ANOVA for the factors category and phase. Therefore, the data from both phases were pooled together and used for the subsequent analyses.

Figure 4 shows the overall mean SR across all subjects, movement features and categories. In general, the recognition of the dynamic stimuli was not an easy task for the subjects. The SRs for the correct identification of all the movement features simultaneously (Fig. 4a) were poor $(66 \pm 15 \%)$. The SRs were similar for simple movements (single lines) and more complex dynamic patterns comprising several segments (geometries and letters), with no statistically significant differences between. Importantly, the subjects were more successful when identifying the movement shape $(77 \pm 13 \%)$, which means that they could interpret the semantic aspect of the 


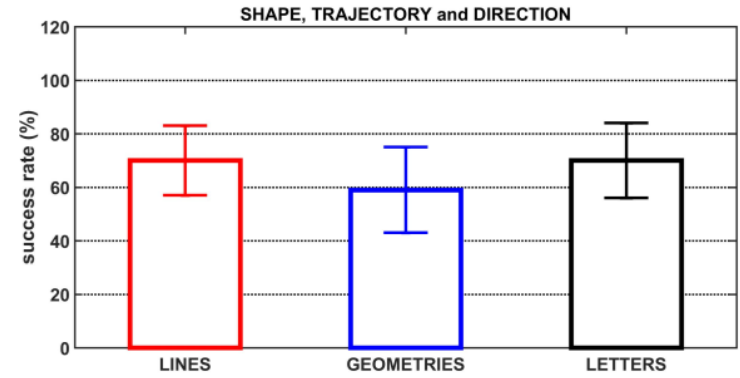

(a)

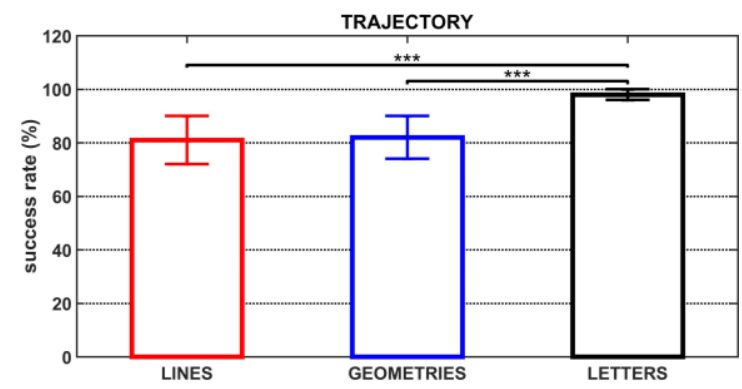

(c)

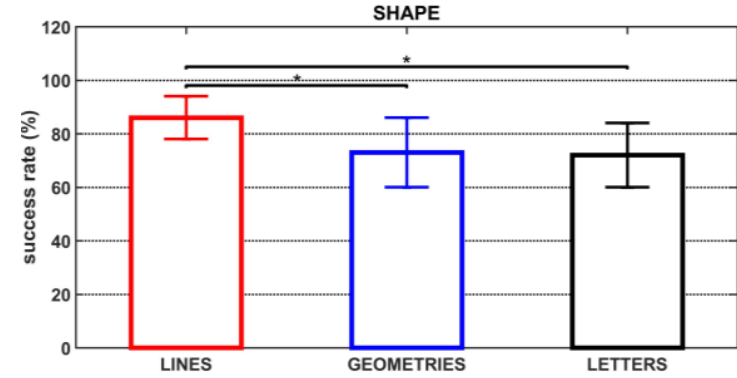

(b)

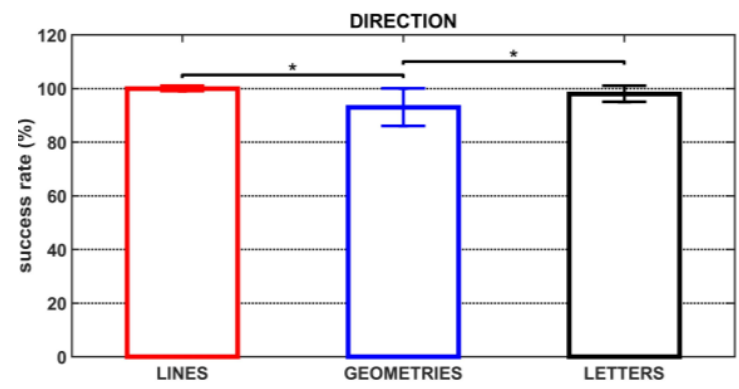

(d)

Fig. 4. Overall success rate of recognizing either (a) all three movement features (shape, trajectory, direction) together, or (b) shape, (c) trajectory and (d) direction provided that the shape has been correctly predicted. This analysis is performed for single lines (RED bars), geometric shapes (BLUE bars) and letters (BLACK bars). Bars and stars indicated statistical significance $\left({ }^{*}, p<0.05 ;{ }^{* *}, p<0.01 ;{ }^{* *}, p<0.001\right)$

movement (e.g., recognize a specific latter or shape). For the movement shape, the performance (Fig. $4 \mathrm{~b}$ ) was better $(\mathrm{p}<0.05)$ for the lines $(86 \pm 8)$ than for the geometries $(73 \pm 13)$ and the letters $(72 \pm 12)$.

When the shape had been properly recognized, the subjects could retrace the full trajectory in $87 \pm 10 \%$ of cases (Fig. 4c), and they could guess the movement direction almost perfectly $(97 \pm 5 \%, \mathrm{p}<0.05)$ (Fig. $4 \mathrm{~d}$ ). Interestingly, the movement trajectory was traced significantly better for the letters $(98 \pm 2 \%)$ compared to the lines $(81 \pm 9 \%, \mathrm{p}<0.001)$ and the geometries $(82 \pm 8 \%, \mathrm{p}<0.001)$, while there were no significant differences between the latter two. Regarding the movement direction, the SR was lower for the geometries $(93 \pm 7)$ than for the lines $(100 \pm 1 \%, p<0.05)$ and the letters $(98 \pm 3 \%, p<0.05)$. The SR for the direction of the lines and the letters was similarly high (no statistical difference).

For the subcategories (transversal, longitudinal, and diagonal) of the line patterns (Fig. 5 - red bars), there was no difference in performance when recognizing the shape (Fig. 5a) and direction (Fig. 5c). In particular, the subjects could identify the direction almost perfectly (SR 100\%). However, the trajectory tracing (Fig. $5 b$ ) was significantly better for the diagonal lines $(99 \pm 4 \%)$ compared to transversal $(79 \pm 14 \%, \mathrm{p}<0.01)$ and longitudinal lines $(76 \pm 13 \%, \mathrm{p}<0.01)$. The three best recognized patterns were the diagonal (drl), transversal ( $\mathrm{t}$ ), and longitudinal (l) line. Overall, the subjects were more successful (Fig. $4 \mathrm{~d}$ vs. c, p
$<0.001)$ in identifying the line direction $(100 \pm 1 \%)$ than in tracing the trajectory $(81 \pm 9 \%)$.

Regarding the geometries (Fig. 5 - blue bars), the movement shape was identified significantly better in triangles $(81 \pm 14 \%)$ and squares $(76 \pm 19 \%)$ compared to rectangles $(56 \pm 19 \%, \mathrm{p}<0.01)$. There was no significant interaction nor main effect in the two-way ANOVA for the factors subcategory and movement feature (trajectory and direction). As for the single lines, direction recognition was easier (Fig. 4c vs. d), i.e., the overall SR was $82 \pm 2 \%$ for trajectory tracing vs. $93 \pm 7 \%$ for direction; but there were no significant differences between the these two. The geometries with the highest SR (Fig. 6b) were triangle (ltr) and square (ls).

The best recognized letters were $Z$, mirrored $Z$ and $L$ (Fig. 6c). Note that those are the letters composed of the best recognized single lines (see Fig. 6a). For the letters, contrary to lines and geometries, there was no statistically significant difference between the trajectory tracing (Fig. 4c, 98 $\pm 2 \%$ ) and identification of direction (Fig. 4 d, $98 \pm 3 \%$ ).

\section{Discussion ANd Conclusion}

A system was implemented to transmit mechanical information from a multipoint tactile sensor (e-skin) to the human subject using multichannel electrotactile stimulation. The system was evaluated by assessing the ability of the human subjects to perceive the properties (shape, trajectory, direction) characterizing dynamic and 


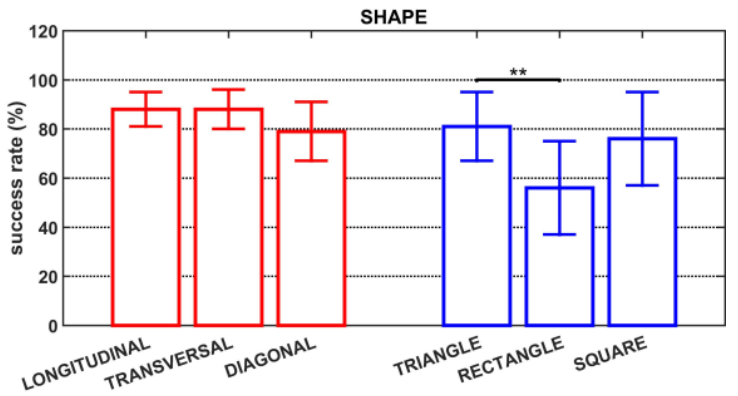

(a)

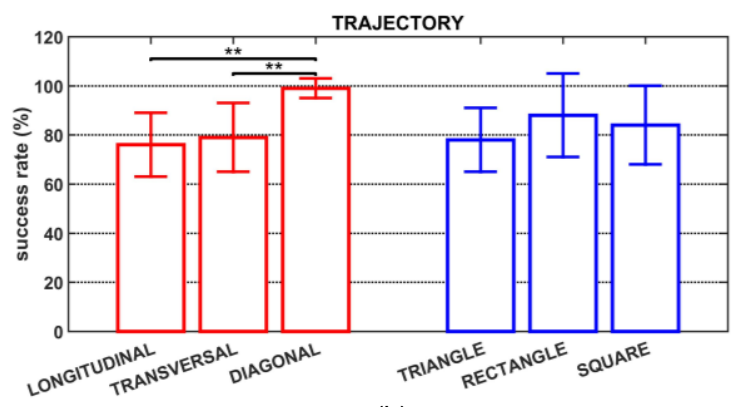

(b)

(c)

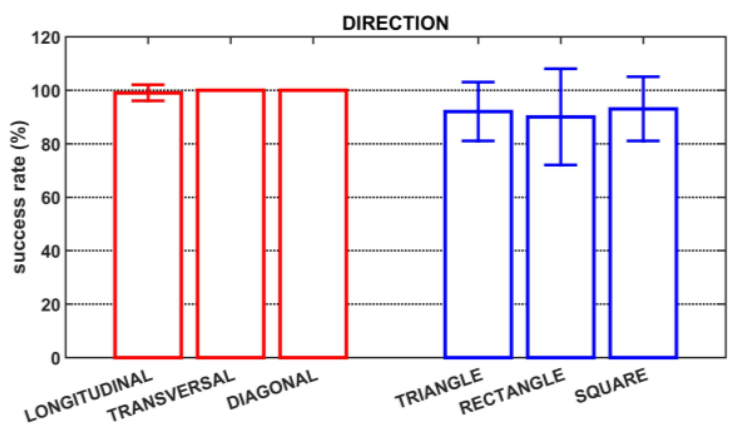

Fig. 5. Success rate of recognizing the shape (a), trajectory (b), and direction (c), for each category of lines (longitudinal, transversal, and diagonal indicated by RED bars) and of geometries (triangle, rectangle and square indicated by BLUE bars). Bars and stars indicated statistical significance $\left({ }^{*}, p<0.05 ;{ }^{* *}, p<0.01 ;{ }^{* * *}, p<0.001\right)$.

versatile (lines, geometries, letters) mechanical interaction with the skin. To the best of our knowledge, this is the first development integrating an advanced tactile sensor with many sensing elements and an electrotactile stimulation unit with a flexible matrix of electrodes into an online system for the transmission of tactile data from artificial to natural skin (forearm).

In [49], the tactile motion patterns were simulated by controlling a $24 \times 6$ matrix of miniature probes indenting the skin. Moving patterns were produced by turning on and off the rows of the matrix in fast succession. The stimulation was delivered to the palm and fingers, since the intended application was the substation of hearing using distributed tactile stimulation. The setup was used in a sequence of studies with microneurography [49]-[51] for the investigation of the basic properties of mechanoreceptors. However, the tactile profiles were preprogrammed, i.e., the setup did not include the sensing element such as the e-skin used in the present study.

The ability of the human subjects to identify the direction of motion over the skin has been investigated in earlier studies [52], [53] to obtain insights into normal and pathological human sensory processing. Typically, an apparatus that could apply well controlled motion of the fine brush over the skin surface was used for the tests. The studies demonstrated that directional sensitivity depends on the body site (mechanoreceptor density), increases with longer trajectories, exhibits preferential range of movement velocities (decreases for too slow and too fast movements), and does not to depend on the movement orientation. The direction sensitivity was tested also by simulating movements using a matrix of tactile stimulators delivering predefined stimulation profiles [54]. The authors concluded that success rate in discriminating directions depends on the number of successively activated stimulation points (amount of sent information), rather than the distance between the first and last point.

The results obtained in the present experiment demonstrate that the novel system successfully translated the tactile signals into profiles of electrical stimulation using the proposed online processing and control. The human subjects could successfully interpret the delivered multichannel stimulation to recognize the properties of tactile stimuli moving across the skin surface. The whole process was running online and thereby under realistic conditions, leading to variability in tactile signals and electrical profiles across the trials, e.g., occasionally activating taxels and electrodes that did not belong to the ideal trajectories (Fig. 6). Importantly, the ability to recognize all the movement features correctly was similar across simple and more complex patterns, indicating robustness. Regarding the shape detection, the subjects could well identify lines with different orientation (longitudinal, transversal, diagonal) with no preference with respect to the specific orientation along the forearm. For more complex patterns, the ability to identify the shape decreased, but the subjects could still recognize quite reliably specific patterns comprising 3 to 4 connected lines (Fig. 6). They could even precisely retrace the full movement trajectory in a reasonable number of cases, and recognize the direction of the movement along the skin with a high confidence. The performance in recognizing direction was consistently better than in trajectory tracing, overall and also for the lines and the geometries. The consistency in identifying the direction is in accordance with the results of previous studies [52]-[54], considering 
(a)

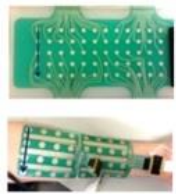

$95 \%$

ti

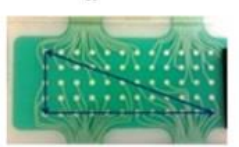

(b)

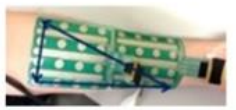

$88 \%$

Itr

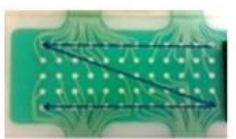

(c)

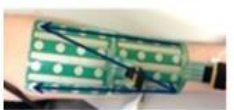

$93 \%$

5

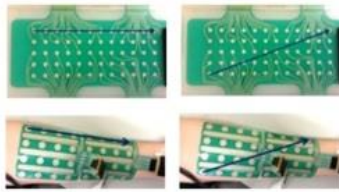

$83 \%$
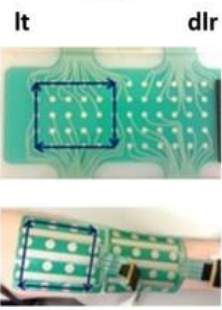

$73 \%$

Is

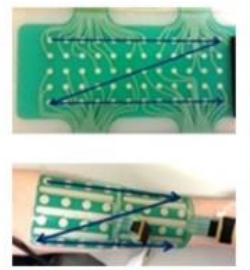

$88 \%$

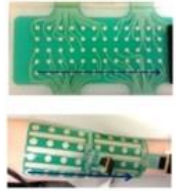

$80 \%$

lb
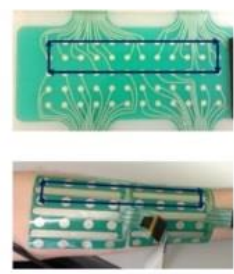

$60 \%$
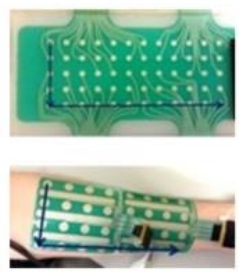

$83 \%$
Fig. 6. Three best recognized patterns: single lines (a), geometries (b), letters (c). For each panel, pictures at the top illustrate how each pattern was applied to the e-skin, pictures below indicate the electrodes activated during electrotactile stimulation to communicate the pattern to the subject, while the success rate and the textual code (Table 1) for the pattern are reported at the bottom.

that our motion patterns were rather long and with multiple stimulation points (e.g., 8 electrode pads for longitudinal lines).

In general, the subjects were more successful with the patterns stretching across the full extent of the e-skin (and thereby matrix electrode) compared to the patterns localized to smaller areas. This is indicated by the fact that the performance was better for the letters than for the geometries in both trajectory tracing and direction identification. Furthermore, the subjects had difficulties when they needed to precisely localize one of several patterns with the same shape but different positions on the forearm. This is a likely reason for the worse performance of trajectory tracing for lines vs. letters, since the former category contains closely spaced longitudinal and transversal lines (Fig. 6). Despite the fact that additional measurements are needed to extend this analysis to a larger number of subjects and investigate the learning effects through training, we can conclude that this sensory feedback system is feasible and intuitive.

There are several possible reasons why the training failed to improve the performance. The training could have been too short and/or dynamic to allow learning. There were many shapes (10 lines, 7 geometries and 8 letters) which were presented randomly and only three times per shape. In addition, there was a level of variability, i.e., tracking the same shape did not evoke exactly the same electrotactile activation, as explained before in this section. Finally, the subjects were not allowed to look at the skin while the experimenter applied the pattern, and therefore he/she received only the tactile information. As demonstrated before [55], multisensory integration might facilitate the learning. All in all, optimal training paradigms for the presented tactile system are yet to be determined.

The tests employed in the present study are similar to those used for assessing graphesthesia [56], [57], which refers to the ability of recognizing numbers traced on the subject own skin. These tests are applied to assess the capability of the tactile sensor system to process differences in the position and direction of a moving tactile input and fuse that information into higher cortical representations. This assessment is conducted in healthy subjects as well as in patients to provide basic insights into the nature of sensory processing and/or diagnostic conclusions. The promising results obtained in the present study demonstrate that the developed skin substitute maintains the consistency of tactile perception and processing. In [56], the subjects recognized 10 numbers (09 , size: $5 \times 7 \mathrm{~cm}$ ) presented by the experimenter moving his index finger along the skin of the dorsal side of the forearm with an SR of $89 \%$. In another study [57], 12 letters were presented by moving a stylus over the palm of the subject resulting in the SR of $75 \%$. In the present study, 8 letters $(9 \times 20 \mathrm{~cm})$ were recognized (shape) with a success rate of approximately $72 \%$. This is in agreement with the previous studies considering that the patterns were delivered using a non-ideal, online loop with electrotactile stimulation (compared to ideal, direct contact with the skin, as used in [56], [57]). The present study demonstrated that the subjects were able to recognize complex shapes, which is an encouraging result. However, the electrical profile was transmitted to the user by sequentially activating the pads along the trajectory. If the profile was presented in parallel, by simultaneously activating all the pads, the recognition would probably worsen [58]. The latter paradigm is likely to be more common during the practical application (e.g., a prosthetic hand grasping an object).

The experimental protocol in the present study was defined by considering the limitations of the available setup and the scope of the current work. These first results are encouraging considering the future applications of the proposed system in prosthetics. The fact that human subjects were able to perceive the tactile features of moving 
electrotactile stimuli can be of relevance for prosthesis control as well as for embodiment. As pointed out in [54], moving tactile stimuli can be an effective method for information transmission to the user, because mechanoreceptors respond stronger to this type of stimuli compared to simple (static) pressure. Also, such perception can be the basis for the exploration of the environment, as when the subject would move the prosthetic hand across the object surface to assess texture by relying on artificial tactile stimulation, as demonstrated through simulations in [59]. Finally, the transmission of a rich, dynamic information between the subject and the prosthesis can be used to promote the embodiment of the artificial system. A similar set of mechanical test patterns, as those used in the present study, could be applied to the prosthesis allowing the subject to perceive, visually and through a tactile channel, a set of realistic interactions. This would be similar to the classic setup for the rubber hand illusion but including a variety of stimulation profiles. The richer stimulation could facilitate the illusion, though this needs to be tested in future experiments.

However, the current study did not yet demonstrate the utility of the proposed technology in the real-life application, which is an important future goal. To this aim, the next step is to cover a myoelectric prosthetic hand with an e-skin in order to test the closed-loop system during functional tasks. The scope is to assess if a sophisticated tactile feedback would increase the utility of the device and/or facilitate the feeling of embodiment. An e-skin covering the prosthetic hand would provide sophisticated tactile sensing that could be used to react to the environment. For example, high sensitivity of the e-skin could be exploited to detect and thereby prevent incipient slip. This could be done automatically, through the prosthesis controller (as in Autograsp feature of Otto Bock Sensor Hand [60]), and/or by transmitting slip feedback to the user, so that he/she can react and manually tighten the grasp (as demonstrated in [61]).

Moreover, contrarily to the passive reception of the electrotactile stimuli, as in the present study, placing the skin on the prosthetic hand would allow the user to actively explore the environment. In this case, the tactile feedback would arise as an interaction between volitional commands and movements of the user and the objects with which he/she interacts. This is known to affect the haptic performance and is therefore an important factor that needs to be considered in the future [62], [63].

Importantly, the e-skin used in the present setup is only an illustrative example of what can be done. The skin production technology is flexible and allows designing patches of different physical characteristics (e.g., size, shape, pad density). For the practical application, the eskin will be dimensioned according to the prosthetic hand to which it will be applied. Ideally, the e-skin should be placed to cover the whole prosthesis, mimicking the skin of the natural hand (as proposed in [64]). We are currently working on the prototype that will be placed on the volar side of the Otto Bock Michelangelo Hand (e.g., finger pads, palm) to support stable grasping.

In addition, data processing and coding will be developed further. This includes the methods for extracting features of the mechanical interaction from the distributed network of highly dynamic sensor elements as well as the translation of that rich, multidimensional information into stimulation patterns that can be interpreted by the subject. The present study represents only an initial step towards exploring these relevant points.

\section{ACKNOWLEDGMENT}

The authors wish to thank SPES MEDICA (Italy) for the realization of the skin prototype, Luigi Pinna (University of Genoa) for the validation of the skin system and Antonio Bozzano for help in collecting data, and Tecnalia Research\&Innovation, Spain, for the MaxSens stimulator and the multi-pad electrodes used in the study.

This work was partly supported by the European Commission projects "ROBOSKIN" under grant agreement no. 231500 and MYOSENS (FP7-PEOPLE-2011IAPP-286208). This work was also supported by Progetto di Ricerca di Ateneo 2014, University of Genova, Italy: Electronic skin for prosthetic systems, resp. M. Valle.

\section{References}

[1] P. Parker, K. Englehart, and B. Hudgins, "Myoelectric signal processing for control of powered limb prostheses.," J. Electromyogr. Kinesiol., vol. 16, no. 6, pp. 541-8, Dec. 2006.

[2] M. Asghari Oskoei and H. Hu, "Myoelectric control systems - A survey," Biomed. Signal Process. Control, vol. 2, no. 4, pp. 275-294, Oct. 2007.

[3] J. C. Rothwell, M. M. Traub, B. L. Day, J. A. Obeso, P. K. Thomas, and C. D. Marsden, "Manual motor performance in a deafferented man.," Brain, vol. 105 (Pt 3), pp. 515-42, Sep. 1982.

[4] J. Ning, S. Dosen, K.-R. Muller, and D. Farina, "Myoelectric Control of Artificial Limbs-Is There a Need to Change Focus? [In the Spotlight]," IEEE Signal Process. Mag., vol. 29, no. 5, pp. 152-150, Sep. 2012.

[5] C. Antfolk, M. D’ Alonzo, B. Rosén, G. Lundborg, F. Sebelius, and C. Cipriani, "Sensory feedback in upper limb prosthetics.," Expert Rev. Med. Devices, vol. 10, no. 1, pp. 45-54, Jan. 2013.

[6] E. Biddiss, D. Beaton, and T. Chau, "Consumer design priorities for upper limb prosthetics.," Disabil. Rehabil. Assist. Technol., vol. 2, no. 6, pp. 346-57, Nov. 2007.

[7] "Vincent Evolution 2 Hand." [Online]. Available: http://vincentsystems.de/en/prosthetics/vincentevolution-2/. [Accessed: 09-Oct-2014]. 
P. Bach-y-Rita and S. W. Kercel, "Sensory substitution and the human-machine interface," Trends Cogn. Sci., vol. 7, no. 12, pp. 541-546, Dec. 2003.

[9] S. Raspopovic, M. Capogrosso, F. M. Petrini, M. Bonizzato, J. Rigosa, G. Di Pino, J. Carpaneto, M. Controzzi, T. Boretius, E. Fernandez, G. Granata, C. M. Oddo, L. Citi, A. L. Ciancio, C. Cipriani, M. C. Carrozza, W. Jensen, E. Guglielmelli, T. Stieglitz, P. M. Rossini, and S. Micera, "Restoring natural sensory feedback in real-time bidirectional hand prostheses.," Sci. Transl. Med., vol. 6, no. 222, p. 222ra19, Feb. 2014

[10] G. A. Tabot, J. F. Dammann, J. A. Berg, F. V Tenore, J. L. Boback, R. J. Vogelstein, and S. J. Bensmaia, "Restoring the sense of touch with a prosthetic hand through a brain interface.," Proc. Natl. Acad. Sci. U. S. A., vol. 110, no. 45, pp. 18279-84, Nov. 2013.

[11] A. Y. Szeto and F. A. Saunders, "Electrocutaneous stimulation for sensory communication in rehabilitation engineering.," IEEE Trans. Biomed. Eng., vol. 29, no. 4, pp. 300-8, Apr. 1982.

[12] K. a Kaczmarek, J. G. Webster, P. Bach-y-Rita, and W. J. Tompkins, "Electrotactile and vibrotactile displays for sensory substitution systems," IEEE Transactions on Biomedical Engineering, vol. 38, no. 1. Ieee, pp. 1-16, 1991.

[13] “Otto Bock Michelangelo Hand," 2014. [Online]. Available: http:/ / www.living-with-michelangelo.com/gb/home/.

[14] BioTAC SynTouch Company, http://www.syntouchllc.com/Products/BioTac/

[15] T. Sekitani, T. Someya, Stretchable, large-area organic electronics, Adv. Mater., 22 (2010), pp. 2228-2246

[16] T. Someya, Y. Kato, T. Sekitani, S. Iba, Y. Noguchi, Y. Murase, H. Kawaguchi, T. Sakurai, Conformable, flexible, large-area networks of pressure and thermal sensors with organic transistor active matrixes, Proc. Natl. Acad. Sci. U.S.A., 102 (August) (2005), pp. 12321-12325

[17] N. Wettels, V. Santos, R. Johansson, G. Loeb, Biomimetic tactile sensor array, Adv. Robot., 22 (2008), pp. 829-849

[18] K. Kim, K.R. Lee, W.H. Kim, K. Park, T. Kim, J. Kim, J.J. Pak, Polymer-based flexible tactile sensor up to $32 \times 32$ arrays integrated with interconnection terminals, Sens. Actuators A: Phys., 156 (December) (2009), pp. 284-291

[19] Y.-J. Yang, M.-Y. Cheng, W.-Y. Chang, L.-C. Tsao, S.-A. Yang, W.-P. Shih, F.-Y. Chang, S.-H. Chang, K.-C. Fan, An integrated flexible temperature and tactile sensing array using PI-copper films, Sens. Actuators A: Phys., 143 (May) (2008), pp. 143-153

[20] C. Lucarotti, C. M. Oddo, N. Vitiello, M. C. Carrozza, Synthetic and Bio-Artificial Tactile Sensing: A Review, Sensors 2013, 13(2), 1435-1466; doi:10.3390/s130201435

[21] R. S. Johansson and K. J. Cole, "Sensory-motor coordination during grasping and manipulative actions.," Curr. Opin. Neurobiol., vol. 2, no. 6, pp. 815-23, Dec. 1992.

[22] E. D. Engeberg and S. Meek, "Enhanced visual feedback for slip prevention with a prosthetic hand.," Prosthet. Orthot. Int., vol. 36, no. 4, pp. 423-9, Dec. 2012.

[23] P. Saccomandi, E. Schena, C. Oddo, L. Zollo, S. Silvestri, and E. Guglielmelli, "Microfabricated Tactile Sensors for Biomedical Applications: A Review," Biosensors, vol. 4, no. 4, pp. 422-448, Nov. 2014

[24] J. Farserotu, J.-D. Decotignie, J. Baborowski, P.-N. Volpe, C. R. Quiros, V. Kopta, C. Enz, S. Lacour, H. Michaud, R. Martuzzi, V. Koch, H. Huang, T. Li, and C. Antfolk, “Tactile prosthetics in WiseSkin," Design, Automation \& Test in Europe Conference \& Exhibition (DATE), 2015. pp. 16951697, 2015.

[25] J. Farserotu, J. Baborowski, J.-D. Decotignie, P. Dallemagne, C. Enz, F. Sebelius, B. Rosen, C. Antfolk, G. Lundborg, A. Bjorkman, T. Knieling, and P. Gulde, "Smart skin for tactile prosthetics," in 2012 6th International Symposium on Medical Information and Communication Technology
[26] M. D'Alonzo, F. Clemente, and C. Cipriani, Vibrotactile stimulation promotes embodiment of an alien hand in amputees with phantom sensations, IEEE Trans. Neural Syst. Rehabil. Eng., vol. 4320, no. c, Jul. 2014.

[27] Tee B. C., Chortos A., Berndt A., Nguyen A. K., Tom A., McGuire A., Lin ZC., Tien K., Bae W.-G., Wang H., Mei P., Chou HH., Cui B., Deisseroth K., Ng TN., Bao Z., A skininspired organic digital mechanoreceptor, Science, (October 2015), Vol. 350 no. 6258 pp. 313-316

[28] C. Pylatiuk, A. Kargov, and S. Schulz, “Design and Evaluation of a Low-Cost Force Feedback System for Myoelectric Prosthetic Hands," JPO J. Prosthetics Orthot., vol. 18, no. 2, pp. 57-61, Apr. 2006.

[29] C. Cipriani, F. Zaccone, S. Micera, and M. C. Carrozza, “On the Shared Control of an EMG-Controlled Prosthetic Hand: Analysis of User-Prosthesis Interaction," IEEE Trans. Robot., vol. 24, no. 1, pp. 170-184, Feb. 2008.

[30] A. Chatterjee, P. Chaubey, J. Martin, and N. Thakor, “Testing a Prosthetic Haptic Feedback Simulator With an Interactive Force Matching Task," JPO J. Prosthetics Orthot., vol. 20, no. 2, pp. 27-34, Apr. 2008.

[31] H. J. B. Witteveen, E. a Droog, J. S. Rietman, and P. H. Veltink, "Vibro- and electrotactile user feedback on hand opening for myoelectric forearm prostheses.," IEEE Trans. Biomed. Eng., vol. 59, no. 8, pp. 2219-26, Aug. 2012.

[32] M. D' Alonzo, S. Dosen, C. Cipriani, and D. Farina, “HyVE Hybrid Vibro-Electrotactile Stimulation - Is an Efficient Approach to Multi-Channel Sensory Feedback," IEEE Trans. Haptics, vol. 7, no. 2, pp. 181-190, Apr. 2014.

[33] I. Saunders and S. Vijayakumar, "The role of feed-forward and feedback processes for closed-loop prosthesis control.," J. Neuroeng. Rehabil., vol. 8, no. 1, p. 60, Jan. 2011.

[34] H.S. Nalwa. Ferroelectric Polymers - Chemistry, Physics and Applications, Marcel Dekker Inc. New York, NY, 1995, pp. 203-214

[35] SPES Medica, Genoa, Italy. Available: http://www.spesmedica.com

[36] Measurement Specialties Inc. Available: http://www.measspec.com/default.aspx

[37] L. Seminara, L. Pinna, M. Valle, L. Basiricò, A. Loi, P. Cosseddu, A. Bonfiglio, A. Ascia, M. Biso, A. Ansaldo, D. Ricci, G. Metta, Piezoelectric polymer transducer arrays for flexible tactile sensors, IEEE Sensors Journal, vol. 13, no. 10, 2013, pp. 4022-4029.

[38] L. Pinna, M. Valle, "Charge Amplifier Design Methodology for PVDF-Based Tactile Sensors," Journal of Circuits, Systems and Computers (JCSC), vol. 22, no. 8, 2013

[39] Štrbac M, Belić M, Isaković M, et al. Integrated and flexible multichannel interface for electrotactile stimulation, J Neural Eng, 2016

[40] Došen S, Marković $M$, Štrbac $M$, et al. Multichannel electrotactile feedback with spatial and mixed coding for closed-loop control of grasping force in hand prostheses. IEEE Trans Neural Syst Rehabil Eng, 2016

[41] Malešević, Nebojša M., et al. "A multi-pad electrode based functional electrical stimulation system for restoration of grasp." Journal of NeuroEngineering andRehabilitation 9.1 (2012): 1.

[42] M. Solomonow, J. Lyman, and A. Freedy, "Electrotactile two-point discrimination as a function of frequency, body site, laterality, and stimulation codes.," Ann. Biomed. Eng., vol. 5, no. 1, pp. 47-60, Mar. 1977.

[43] Minja at al. "Electrical stimulation of the forearm: a method for transmitting sensory signals from the artificial hand to the brain" Journal of automatic control, vol. 21, no. 1, pp 1318, 2013.

[44] Lee, Stephen; Kruse, John. "Biopotential Electrode Sensors in ECG/EEG/EMG Systems", Analog Devices, 2014

[45] B. Geng, K. Yoshida, L. Petrini, and W. Jensen, “Evaluation information. 
of sensation evoked by electrocutaneous stimulation on forearm in nondisabled subjects," J. Rehabil. Res. Dev., vol. 49, no. 2, p. 297, 2012.

[46] T. Tashiro and A. Higashiyama, "The perceptual properties of electrocutaneous stimulation: Sensory quality, subjective intensity, and intensity-duration relation," Percept. Psychophys., vol. 30, no. 6, pp. 579-586, Nov. 1981.

[47] L. P. Paredes, S. Dosen, F. Rattay, B. Graimann, and D. Farina, "The impact of the stimulation frequency on closedloop control with electrotactile feedback.," J. Neuroeng. Rehabil., vol. 12, no. 1, p. 35, Jan. 2015.

[48] F. A. A. Kingdom and N. Prins, Psychophysics: A Practical Introduction. Academic Press, 2009.

[49] E. P. Gardner and C. I. Palmer, "Simulation of motion on the skin. I. Receptive fields and temporal frequency coding by cutaneous mechanoreceptors of OPTACON pulses delivered to the hand.," J. Neurophysiol., vol. 62, no. 6, pp. 1410-36, Dec. 1989.

[50] E. P. Gardner and C. I. Palmer, "Simulation of motion on the skin. III. Mechanisms used by rapidly adapting cutaneous mechanoreceptors in the primate hand for spatiotemporal resolution and two-point discrimination.," J. Neurophysiol., vol. 63, no. 4, pp. 841-59, Apr. 1990.

[51] E. P. Gardner and C. I. Palmer, "Simulation of motion on the skin. II. Cutaneous mechanoreceptor coding of the width and texture of bar patterns displaced across the OPTACON.," J. Neurophysiol., vol. 62, no. 6, pp. 1437-60, Dec. 1989.

[52] D. A. Dreyer, M. Hollins, and B. L. Whitsel, "Factors influencing cutaneous directional sensitivity.," Sens. Processes, vol. 2, no. 2, pp. 71-9, Jun. 1978.

[53] G. K. Essick and B. L. Whitsel, "Factors influencing cutaneous directional sensitivity: a correlative psychophysical and neurophysiological investigation.," Brain Res., vol. 357, no. 3, pp. 213-30, Dec. 1985.

[54] E. P. Gardner and B. F. Sklar, “Discrimination of the direction of motion on the human hand: a psychophysical study of stimulation parameters.," J. Neurophysiol., vol. 71, no. 6, pp. 2414-29, Jun. 1994.

[55] Shams, L., \& Seitz, A. R. (2008). Benefits of multisensory learning. Trends in cognitive sciences, 12(11), 411-417.

[56] C. D. Mørch, O. K. Andersen, A. S. Quevedo, L. ArendtNielsen, and R. C. Coghill, "Exteroceptive aspects of nociception: insights from graphesthesia and two-point discrimination.," Pain, vol. 151, no. 1, pp. 45-52, Oct. 2010.

[57] V. Drago, P. S. Foster, D. Edward, B. Wargovich, And K. M. Heilman "Graphesthesia: A test of graphemic movement representations or tactile imagery?," J. Int. Neuropsychol. Soc., vol. 16, no. 01, p. 190, Oct. 2009.

[58] Loomis, J. M., \& Lederman, S. J. (1986). Tactual perception. Handbook of perception and human performances, 2, 2

[59] C. E. Kops and E. P. Gardner, "Discrimination of simulated texture patterns on the human hand.," J. Neurophysiol., vol. 76, no. 2, pp. 1145-65, Aug. 1996.

[60] "Sensor Hand." [Online]. Available: http:/ / www.ottobock.com.au/prosthetics/upper-

limb/solution-overview/myoeletric-devices-speedhands/.

[61] D. D. Damian, A. H. Arita, H. Martinez, and R. Pfeifer, "Slip Speed Feedback for Grip Force Control," IEEE Trans. Biomed. Eng., vol. 59, no. 8, pp. 2200-2210, Aug. 2012.

[62] R. L. Klatzky and S. J. Lederman, Touch. Handbook of Psychology. Two: 6, pp. 147-176, 2003.

[63] S. J. Lederman \& R. L. Klatzky. Hand movements: A window into haptic object recognition. Cognitive psychology, vol. 19, no. 3, pp. 342-368, 1987.

[64] J. Kim, M. Lee, H. J. Shim, R. Ghaffari, H. R. Cho, D. Son, Y. H. Jung, M. Soh, C. Choi, S. Jung, K. Chu, D. Jeon, S.-T. Lee, J. H. Kim, S. H. Choi, T. Hyeon, and D.-H. Kim, “Stretchable silicon nanoribbon electronics for skin prosthesis," Nat. Commun., vol. 5, p. 5747, Dec. 2014.

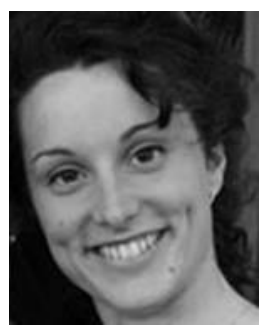

Marta Franceschi received her BS degree at the University of Genoa (IT) in 2013. In 2015 she obtained her MS degree in Electronic Engineering at the University of Genoa (IT). From June to December 2015 she was DAAD student at the Institute of Neurorehabilitation Systems of the University Medical Center Goettingen, being involved in developing of a prosthetic tactile feedback system based on artificial skin and electrotactile stimulation. Currently, she is $\mathrm{PhD}$ student in Science and Technology for Electronic and Telecommunication Engineering.

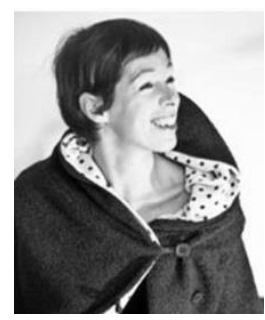

Lucia Seminara was born in London (UK) in 1974 and obtained her MS degree in Physics at the University of Genoa (IT) in 1999. In 2004 she obtained her PhD degree in Physics at the EPFL in Lausanne (CH). From 2005 to 2009 she worked in a Telecommunications Company, working on wireless networks of nanostructured gas sensors. Since 2009 she works as a post-doctoral researcher at the Department of Naval, Electrical, Electronic and Telecommunications Engineering (DITEN) of the University of Genoa, being involved in materials science aspects and processing of the tactile information related to the development of an electronic skin based on arrays of piezoelectric polymer sensors. She is IEEE member of the IEEE Engineering in Medicine and Biology Society.

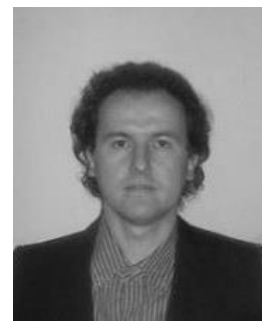

Strahinja Dosen (M'05) received the Diploma of Engineering in electrical engineering and the M.Sc. degree in biomedical engineering in 2000 and 2004, respectively, from the Faculty of Technical Sciences, University of Novi Sad, Serbia, and the Ph.D. degree in biomedical engineering from the Center for Sensory-Motor Interaction, Aalborg University, Aalborg, Denmark, in 2008. Until 2011, Dr. Dosen was an Assistant Professor at the same Department. Currently, he is a Research Scientist at the Institute for Neurorehabilitation Systems, University Medical Center Göttingen (UMG), Germany. His main research interest is in the closed-loop control of movements and assistive systems, including prosthesis control, sensory feedback, functional electrical stimulation, and rehabilitation robotics. He is a member of the IEEE Engineering in Medicine and Biology Society and the IEEE Computer Society.

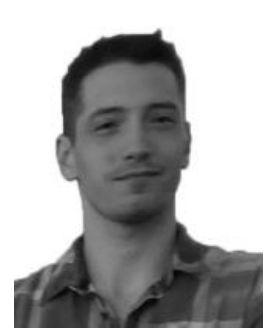

Matija Štrbac received BSc and MSc degrees from the University of Belgrade - School of Electrical Engineering in 2010 and 2011, respectively. In 2011 he enrolled PhD studies at the same faculty with the focus on development of the artificial perception system that enables automatic control of electrical stimulation. From January 2012 until December 2015 Matija was a research assistant at the School of Electrical Engineering and a collaborator of the project of the Ministry of Education, Science and Technological Development of the Republic of Serbia. From April 2013 he started working for Tecnalia Serbia Ltd. on the development of the electrotactile feedback interface for myoelectric hand prostheses.

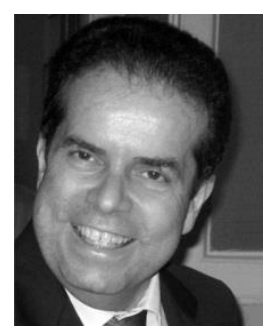

Maurizio Valle received the M.S. degree in Electronic Engineering in 1985 and the Ph.D. degree in Electronic and Computer Science Engineering in 1990 from the University of Genova, Italy. Since January 2007, he is associate professor in Electronic Engineering at Department of Electrical, Electronic and Telecommunications Engineering and Naval Architecture, University of Genova. Maurizio Valle has been and is in charge of many research contracts and projects funded at local, national and European levels and by Italian and foreign companies. Among others, he has been guest editor of the Special Issue on Robotic Sense of Touch, IEEE Transactions on Robotics, 2011; Guest Editor, Special Issue "Tactile Sensors and Sensing Systems", in Sensors, MDPI - Open Access Publishing, 2013. He is co-author of more than 200 papers on international scientific journals and conference proceedings and of the book Robotic Tactile Sensing - Technologies and System, Springer Science + Business Media, 2013. His research interests include materials integrated sensing systems, electronic tactile sensors and 
This article has been accepted for publication in a future issue of this journal, but has not been fully edited. Content may change prior to final publication. Citation information: DOI 10.1109/TOH.2016.2618377, IEEE Transactions on Haptics

systems, microelectronic embedded systems, wireless sensors networks. He is IEEE member.

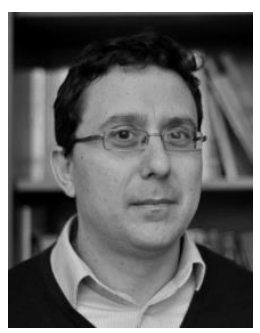

Dario Farina received the M.Sc. degree in electronics engineering from Politecnico di Torino, Torino, Italy, in 1998, and the Ph.D. degree in automatic control and computer science and in electronics and communications engineering from the Ecole Centrale de Nantes, Nantes, France, and Politecnico di Torino in 2001. From 1997 to 2004 he was a researcher at the Laboratory for Neuromuscular System Engineering (LISiN) of Politecnico di Torino. During 2004-2008 he was an Associate Professor in Biomedical Engineering at Aalborg University, Aalborg, Denmark. At the same University, in 2008 he became Full Professor in Motor Control and Biomedical Signal Processing and the Head of the Research Group on Neural Engineering and Neurophysiology of Movement. In 2010 he was appointed Full Professor and Founding Chair of the Department of Neurorehabilitation Engineering at the University Medical Center Göttingen, Georg-August University, Germany, within the Bernstein Focus Neurotechnology (BFNT) Göttingen. Prof. Farina has been the President of the International Society of Electrophysiology and Kinesiology (ISEK) in 20122014 and currently holds the position of Past President. His research focuses on neurorehabilitation technology, neural control of movement, and biomedical signal processing and modelling. Within these areas, Prof. Farina has (co)authored more than 350 papers in peer-reviewed Journals and over 400 among conference papers/abstracts, book chapters, and encyclopedia contributions. 